\title{
Response to Theta-Burst rTMS in Treatment-Resistant Depression (A Case Report)
}

\author{
Marachev M.P. ${ }^{1}$, Grigoryeva A.A. ${ }^{1}$, Staroverov I.I. ${ }^{2}$, Alfimov P.V. ${ }^{3}$
}

1 Neurocenter of medical and psychological correction and rehabilitation

2 Lomonosov Moscow State University Faculty of Medicine;

3 Moscow Scientific Research Institute of Psychiatry Branch of V. Serbsky National Medical Research Centre for Psychiatry and Narcology, Moscow, Russia (No potential conflict of interest)

\section{Introduction}

Repetitive transcranial magnetic stimulation (rTMS) is a promising option for patients with treatment-resistant depression (TRD). Reported response rates for rTMS in TRD are $45-60 \%$. A high rate of non-responders implicates a need for new treatment strategies. Those may include alternative rTMS protocols, e.g. theta-burst stimulation (TBS), use of different coils, etc.

\section{Purpose}

We are reporting a case of a highly-resistant patient with recurrent depression, a 40-yearold male. Previously, he received adequate pharmacological treatment, and bilateral ECT. The treatment had minor-to-moderate and unsustainable effect. Cognitive disfunction was the most subjectively impairing symptom. The patient responded well to TBS.

\section{Methods}

\section{TBS}

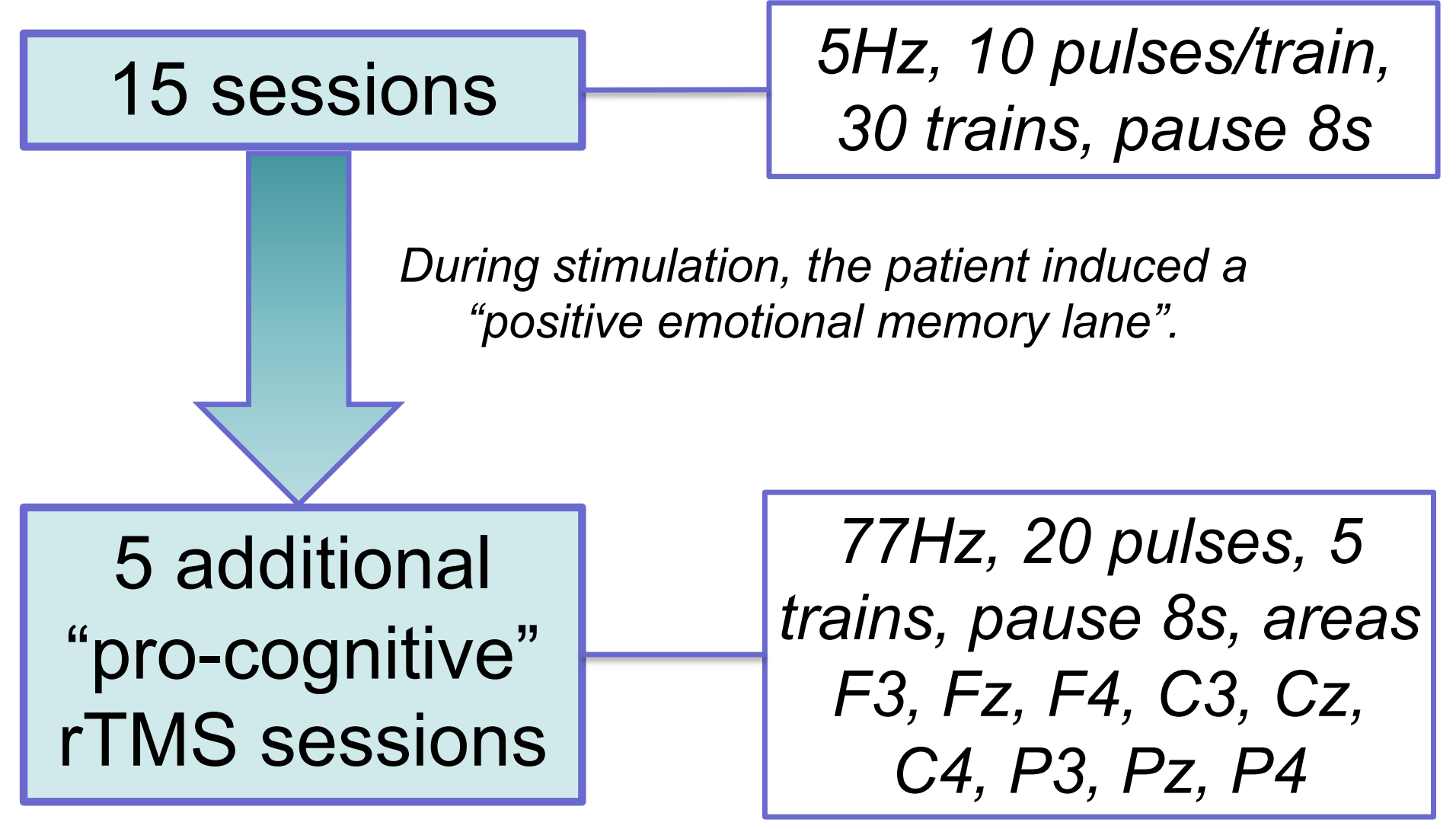

$B D I$ and BAI scales were used for assessment

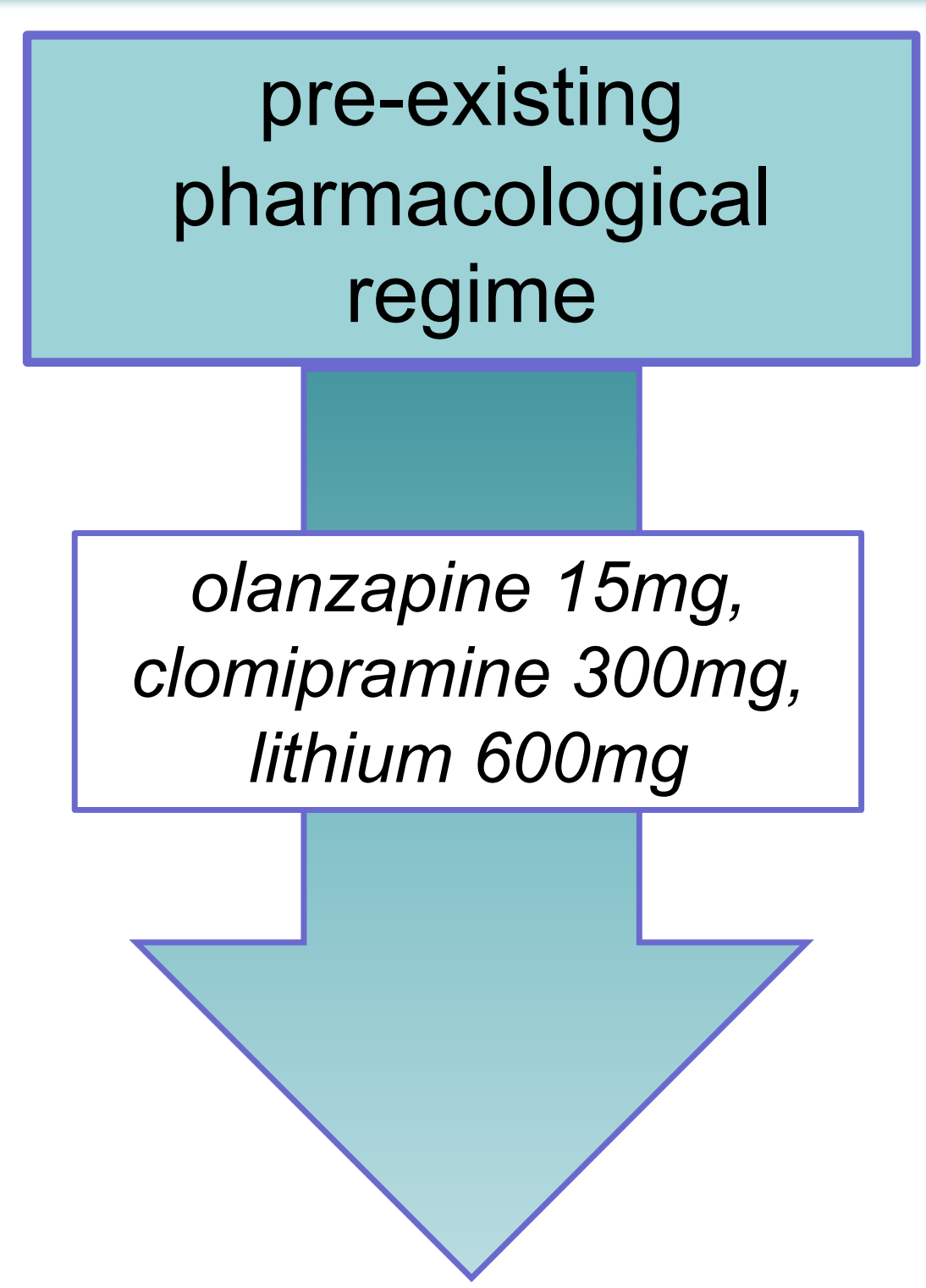

Conclusion.

An intensive and varying brainstimulation approach may be successful in super-refractory depressed patients with "exhausted" pharmacological options.

\begin{tabular}{|c|c|c|c|}
\hline \multicolumn{4}{|c|}{ Results } \\
\hline & BDI & BAI & $\begin{array}{l}\text { SUBJECTIVE } \\
\text { SELF-REPORT }\end{array}$ \\
\hline $\begin{array}{l}\text { INITIAL } \\
\text { LEVEL }\end{array}$ & 49 & 26 & - \\
\hline AFTER 4 TBS & 34 & 13 & $50 \%$ improvement \\
\hline $\begin{array}{c}\text { AFTER } 15 \\
\text { TBS }\end{array}$ & 25 & 6 & $\begin{array}{c}\text { Persistence of } \\
\text { anxiety and } \\
\text { cognitive } \\
\text { disfunction }\end{array}$ \\
\hline $\begin{array}{l}\text { ADDITIONAL } \\
5 \text { TBS }\end{array}$ & - & - & $\begin{array}{l}\text { Remission with } \\
\text { good functioning } \\
\text { and quality of life }\end{array}$ \\
\hline
\end{tabular}

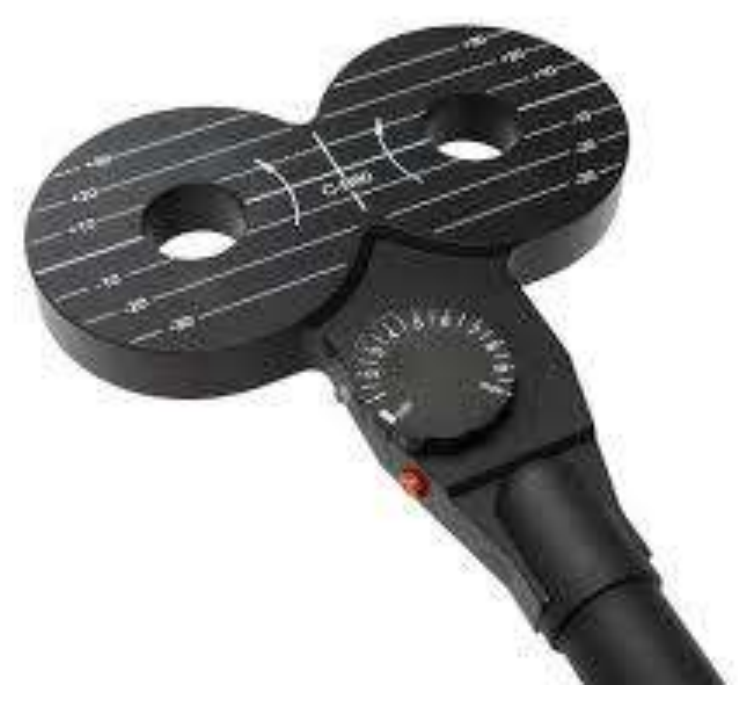

